\title{
Harmonic Internal Models for Structurally Robust Periodic Output Regulation
}

\author{
Daniele Astolfi ${ }^{\mathrm{a}}$, Laurent Praly ${ }^{\mathrm{b}}$, Lorenzo Marconi $^{\mathrm{c}}$ \\ ${ }^{a}$ Univ Lyon, Université Claude Bernard Lyon 1, CNRS, LAGEPP UMR 5007, 43 boulevard du 11 novembre 1918, F-69100, Villeurbanne, France. \\ ${ }^{b}$ Centre Automatique et Systèmes, MINES ParisTech, Université PSL, Paris, France \\ ${ }^{c}$ CASY-DEI, University of Bologna, Bologna 40123, Italy
}

\begin{abstract}
This note deals with the problem of output regulation for nonlinear systems in presence of periodic exogenous signals. We investigate the asymptotic properties of a controller given by an internal model designed by adding harmonics on the regulation error, and a static state feedback stabilizing the augmented system of the plant and of the internal model. The solution mimics internal model-based structures adopted for linear systems by showing the asymptotic properties that are guaranteed in the nonlinear case in presence of "generic" plant variations. Forwarding technique is adopted in the design of the stabilizer. We shed also light on the linear case by presenting a new easy-to-check condition under which the regulator equations admit a robust solution.
\end{abstract}

Keywords: Robust regulation, Nonlinear Control, Forwarding, Uncertain dynamic systems

\section{Introduction}

The problem of tracking desired references while rejecting disturbances in spite of model uncertainties is generically known as robust output regulation. In this context, the exogenous variables (i.e. the references and the disturbances) are usually supposed to be generated by a known autonomous system. In the context of linear dynamics such a problem was independently addressed and solved around the '70s in the set of works [1], [2], in which the so-called internal model principle was introduced. The principle states that the problem is solved as long as the regulator "incorporates a suitably reduplicated model of the dynamic structure of the disturbance and reference signals". The solution to the problems lies therefore in the design of a regulator composed of two components: an internal model unit, containing a copy of the model of the exosystem, and a stabilizer unit selected so that to guarantee overall closed-loop stability.

Preliminary and consistent results in nonlinear output regulation were early made in [3] where necessary conditions were studied. The problem can be formalized in the context of nonlinear systems of the form

$$
\begin{aligned}
\dot{x} & =\tilde{f}(x, w, u), \\
e & =\tilde{h}(x, w, u)
\end{aligned}
$$

where $x \in \mathbb{R}^{n}$ is the state, $u \in \mathbb{R}^{m}$ the control input, $e \in \mathbb{R}^{p}$ is the output to be regulated, and $w \in \mathbb{R}^{\rho}$ represents the exogenous variables. The anchor point of the discussion is the solution of the regulator equations, that is, a trajectory, possibly not unique, $\left(w(t), \pi_{x}(t), \psi(t)\right)$ fulfilling

$$
\dot{\pi}_{x}(t)=\tilde{f}\left(\pi_{x}(t), w(t), \varphi(t)\right) \quad 0=\tilde{h}\left(\pi_{x}(t), w(t), \psi(t)\right)
$$

Email addresses: daniele.astolfi@univ-lyon1.fr (Daniele Astolfi), laurent .praly@mines-paristech.fr (Laurent Praly), lorenzo-marconi@unibo.it (Lorenzo Marconi) for all $t \geq 0$. Such regulator equations describe the desired asymptotic behavior of the system (1) on which the regulated output is constantly zero. The function $\pi_{x}$ represents the steadystate for the state $x$ and while $\psi$ is the steady-state for the control input $u$. The relevance of these equations in a general "nonequilibrium" output regulation context was studied in [4]. The main difficulties in the regulator design lie in the intrinsic interdependency between the internal model unit (intuitively responsible for the generation of the steady-state control input $\psi(t)$ ) and the stabilizing unit (intuitively having the role of making the trajectory $\pi_{x}(t)$ attractive), whose design, unlike the linear case, can be hardly kept disjoint and accomplished in separate design stages. We recall that a crucial observation in such a context, early made for instance in [5], is the fact that in a general nonlinear context, the internal-model unit needs to incorporate more dynamics than the one generated by the exosystem because of nonlinear deformation phenomenon. The design of a nonlinear stabilizer itself may contribute to such a phenomenon. These difficulties lead to a "chicken-egg dilemma" highlighted in [6] that makes the design of the units intertwined and hard to be accomplished in practice. The problem is also particularly evident when there are measurements available (and sometimes needed) for stabilization that are not vanishing in steady-state ([7]), as in a state feedback scenario. This justified why most of the contributions in literature consider error feedback solutions in which the stabilizer, having only $e$ as available measurement, has the origin as a natural steady-state. In these scenarios, the design of the two units can be decoupled. Typical design approaches follow a "friend-centric" perspective, namely the internal model unit is tailored by assuming that $\psi(t)$, often referred to as the "friend" of $\pi_{x}(t)$, falls into a specific class of signals, and making "immersion" assumptions asking that the signals in that class are generated as output of a dynamic system (which is used as core of the internal model unit) fulfilling 
some observability properties (see, e.g., [8], [9], [10]). The internal model unit, placed on the input of the regulated plant, then originates an extended system that, written in appropriate error coordinates, lends itself to be stabilized at the origin by an error feedback stabilizing unit. In this respect, high-gain design techniques ([8]) and KKL observers ([9]) have been shown to be effective for the class of regulated plants that are minimumphase with respect to the error. Semi-global [9, 8] or global $[11,12,13]$ results can be obtained for minimum-phase systems with incrementally stable inverse dynamics, based on combinations of high-gain arguments, backstepping techniques or passivity.

The robustness issue is typically dealt with by assuming that variations in the actual process reflect into "fluctuations" of the friend within the same class of signals that, in turn, are mapped in appropriate structured parameterizations of the internal model unit $([14,15])$. This friend-centric perspective, however, leads to "fragile" regulators that are not able to handle "generic" variations in the plant. Generic unstructured variations in the plant, in fact, could reflect in substantial changes on the friend exiting the class around which the internal model unit is shaped and, thus, loosing the internal model property. As conjectured in $[16,17]$ a finite dimensional nonlinear regulator that is able to achieve asymptotic regulation in face of generic plant variations doesn't exist and practical, rather than asymptotic, regulation is claimed to be the right target. In $[16,17]$, in particular, it is introduced a new topological definition of robustness, which is linked to desired asymptotic properties to be enforced in face of plant variations within the fixed topology. Such desired asymptotic proprerties can be potentially different from the more-standard objective "regulation error asymptotically vanishing". Along these lines, an attempt to design robust practical regulators handling "generic" uncertainties can be found in [18]. As for the cases in which measurements not vanishing in steady-state are available, the literature is definitely much less rich. A recent attempt was done in [7] in which a design procedure based on "washing out" the steady-state of the non-vanishing measurements to enforce a zero steady-state in the stabilizer was proposed. The washout filter, however, still relies on immersion assumptions of the same kind recalled before and thus the asymptotic properties are easily lost in case of "generic" plant variations.

\subsection{Objectives and contributions}

This article aims to explore a design methodology that is not tailored around a specific "friend" so that to decouple the design of the internal-model from the stabilizer. We focus on the case in which the exogenous signal $w \in \mathbb{R}^{\rho}$ is not supposed to be generated by an exosystem. Instead, it is supposed that it is any bounded $C^{1} T$-periodic signal, with $T$ being known. In the spirit of $[19,20]$, the ideal, but fragile, property "regulation error asymptotically vanishing" is replaced by the property "Fourier coefficients linked to the frequencies copied in the internal model are canceled on the asymptotic error" that is however preserved without hard restrictions on topologies governing the plant variations. As shown in many applications, e.g.,
$[21,19,22,23]$ and references therein, such a desirable property is often satisfactory from a practical point of view.

The targeted regulator follows the design principle "add harmonics on the regulation error and stabilize the extended system", namely consists of a linear internal model unit obtained by simply embedding an harmonic at the corresponding frequency $\frac{2 \pi}{T}$ of the periodic signal $w$ and a certain number of higher order harmonics, and of a nonlinear stabilizing unit which is designed following the so-called forwarding technique introduced for the stabilization of cascade systems in the 90's, see, e.g. [24] [25] and references therein. It is shown that in presence of periodic exosignals $w$ of "small" magnitude, the closed-loop system trajectories converge to a periodic steadystate on which the desired harmonic regulation objective is obtained. The domain of attraction of such a periodic steady-state is semi-global in the set of initial conditions of the plant. Such a property is also robust to (small) arbitrarily variations of the plant's dynamics. It is worth noting that this work can be seen as an extension of principles introduced [25] where the simpler case of constant perturbations were addressed, combined with our preliminary conference result [20].

The proposed approach is then specialized to the class of bilinear systems. It is worth recalling that despite many engineering applications of practical interest can be modeled with bilinear dynamics (see, e.g., [26, 27]), the literature on output regulation for such a class of systems is quite scarce. To the best of the author's knowledge, only the case of constant disturbances has been partially investigated (see, e.g. [28]) while most of existing approaches cannot include these systems in their theory because the relative degree is generically not defined and a canonical normal form cannot be obtained (see, e.g. $[9,8])$.

Finally as a by-product of the proposed forwarding-based framework, we also present a new easy-to-check condition for the existence of the linear regulator equations which is equivalent to the standard non-resonance condition. We recall that the non-resonance condition is shown to be necessary for the existence of a solution to the linear robust output regulation problem $[1,2]$.

The rest of the paper is organized as follows. In Section 2, we formalize the nonlinear problem we aim at addressing and the main result concerning the design of the regulator is presented. New insights in linear output regulation, resulting as a byproduct of the tools employed in the nonlinear setting, are given in Section 3. The special case of bilinear systems is addressed in Section 4 as illustration. Conclusions are drawn in Section 5

\section{Main Results}

Consider a multi-input multi-output nonlinear system with nominal dynamics taking the form

$$
\begin{aligned}
\dot{x} & =f(x, w)+g(x, w) u \\
e & =h(x, w)
\end{aligned}
$$

where $x \in \mathbb{R}^{n}, u \in \mathbb{R}^{m}$, $e \in \mathbb{R}^{p}$. We consider the particular, yet relevant, case in which the exogenous signal $w \in \mathbb{R}^{\rho}$ is 
any bounded $C^{1} T$-periodic signal, with $T$ being known. The following assumptions are made.

Assumption 1 (Stabilizability) There exists a $C^{1}$ function $\alpha$ : $\mathbb{R}^{n} \rightarrow \mathbb{R}^{m}, \alpha(0)=0$, such that the system $\dot{x}=f(x, 0)+$ $g(x, 0) \alpha(x)$ is asymptotically and locally exponentially stable with domain of attraction an open set $\mathcal{A} \subseteq \mathbb{R}^{n}$.

Assumption 2 (Non-resonance condition) There exists a positive integer $v>0$ so that following matrix

$$
\left(\begin{array}{cc}
A-\lambda I & B \\
C & 0
\end{array}\right)
$$

has independent rows for each $\lambda=i k \frac{2 \pi}{T}, k \in\{0,1, \ldots, v\}$, with the triplet $(A, B, C)$ defined as

$$
A:=\frac{\partial f}{\partial x}(0,0), \quad B:=g(0,0), \quad C:=\frac{\partial h}{\partial x}(0,0) .
$$

Assumption 1 asks for the existence of a stabilizer for the origin of the nominal system (2) in absence of perturbations, with some desired domain of attraction $\mathcal{A}$. In the linear context such an assumption simply coincides with the stabilizability of the system (see below in Section 3) and it is shown to be necessary $[1,2]$. For nonlinear systems, it can be obtained via different techniques (e.g., high-gain feedback, backstepping, forwarding, passivity, Lyapunov-based) for which we will now enter in the merit.

The second Assumption 2 ask for the standard non-resonance condition to hold, although only locally around the origin. Again, such an assumption is shown to be necessary in the linear context $[1,2]$.

By following the paradigm "add harmonics on the regulation error and stabilize the extended system", the internal model unit is immediately chosen as

$$
\dot{\xi}=\Phi \xi+\Gamma e
$$

where $\xi=\operatorname{col}\left(\xi_{1}, \ldots, \xi_{p}\right), \xi_{k} \in \mathbb{R}^{1+2 v}, k=1, \ldots, p$,

$$
\Phi=\operatorname{blkdiag}(\underbrace{\phi, \ldots, \phi}_{p \text { times }}), \Gamma=\operatorname{blkdiag}(\underbrace{G, \ldots, G}_{p \text { times }}),
$$

with

$$
\phi=\operatorname{blkdiag}\left(0, \phi_{1}, \ldots, \phi_{\rho}\right), \phi_{k}=\left(\begin{array}{cc}
0 & k \frac{2 \pi}{T} \\
-k \frac{2 \pi}{T} & 0
\end{array}\right),
$$

and $G=\operatorname{col}(\gamma, \bar{G}, \ldots \bar{G})$, with $\gamma$ a positive scalar and $\bar{G} \in \mathbb{R}^{2 \times 1}$ chosen so that the pairs $\left(\phi_{k}, \bar{G}\right), k=1, \ldots, v$, are controllable. Without loss of generality we can take $\gamma=1$ and $\bar{G}=\left(\begin{array}{ll}0 & 1\end{array}\right)^{\top}$. The following result shows a first preliminary property of a regulator having the structure (4) in closed-loop with a statefeedback stabilizing unit of the form

$$
u=K(x, \xi)
$$

Proposition 1 Suppose there exists a $C^{1}$ function $K: \mathbb{R}^{n} \times$ $\mathbb{R}^{(2 v+1) p} \rightarrow \mathbb{R}^{m}$ such that system (2), (4), (5) in closed loop with (6), with $w$ being a bounded $C^{1} T$-periodic function, admits a $C^{1} T$-periodic solution $\left(x^{\circ}, \xi^{\circ}\right)$. Then, this periodic solution is such that the Fourier coefficients of $e^{\circ}=h\left(x^{\circ}, w\right)$ associated to the frequencies $2 k \pi / T$, with $k=0,1, \ldots, v$, are zero, namely

$$
0=\int_{0}^{T}\left(\begin{array}{c}
\sin \left(k \frac{2 \pi}{T} t\right) \\
\cos \left(k \frac{2 \pi}{T} t\right)
\end{array}\right) e_{i}^{\circ}(t) d t \quad \begin{aligned}
& i=1, \ldots, p, \\
& k=0,1, \ldots, \nu .
\end{aligned}
$$

Proof: The result comes from the fact that the internal model unit is linear and embeds the exosystem frequencies. As a matter of fact, the periodic trajectory fulfills

$$
\dot{\xi}_{i}^{\circ}=\phi \xi_{i}^{\circ}+G e_{i}^{\circ}
$$

for $i=1, \ldots, p$, and, by letting $\xi_{i}=\operatorname{col}\left(\xi_{i 0}, \xi_{i 1}, \ldots, \xi_{i v}\right)$ and using the block diagonal structure of $\phi$, the different components of $\xi_{i}$ fulfill

$$
\begin{aligned}
\dot{\xi}_{i 0}^{\circ} & =\gamma e_{i}^{\circ}, \\
\dot{\xi}_{i k}^{\circ} & =\phi_{k} \xi_{i 0}^{\circ}+\bar{G} e_{i}^{\circ}, \quad k=1, \ldots, v .
\end{aligned}
$$

Integration of the first component yields

$$
\xi_{i 0}^{\circ}(T)=\xi_{i 0}^{\circ}(0)+\gamma \int_{0}^{T} e_{i}^{\circ}(t) d t
$$

which, by using $T$-periodicity of $\xi_{i 0}^{\circ}(t)$ and the fact that $\gamma$ is not zero, implies

$$
\int_{0}^{T} e_{i}^{\circ}(s) d s=0
$$

namely the first Fourier coefficient of $e_{i}^{o}(t)$ is zero, i.e. it possesses a zero DC-component. As for the other components, integration of the generic $k$-th component yields

$$
\xi_{i k}^{\circ}(T)=\exp \left(\phi_{k} T\right) \xi_{i k}^{\circ}(0)+\int_{0}^{T} \exp \left(\phi_{k}(T-t)\right) \bar{G} e_{i}^{\circ}(t) d t .
$$

Observing that

$$
\exp \left(\phi_{k} t\right)=\left(\begin{array}{cc}
\cos \left(k \frac{2 \pi}{T} t\right) & \sin \left(k \frac{2 \pi}{T} t\right) \\
-\sin \left(k \frac{2 \pi}{T} t\right) & \cos \left(k \frac{2 \pi}{T} t\right)
\end{array}\right),
$$

which in particular implies that $\exp \left(\phi_{k} T\right)=I$, and using the fact that $\xi_{i 0}^{\circ}(t)$ is $T$-periodic and the definition of $\bar{G}$, yields

$$
\int_{0}^{T}\left(\begin{array}{c}
\sin \left(k \frac{2 \pi}{T} t\right) \\
\cos \left(k \frac{2 \pi}{T} t\right)
\end{array}\right) e_{i}^{\circ}(t) d t=0
$$

from which the claim of the proposition follows.

Motivated by the previous proposition we look now for a control law (6) able to enforce a $T$-periodic trajectory that is locally asymptotically stable. This, in turn, will guarantee that the closed-loop trajectory will reach a steady-state in which the first $v$ Fourier coefficients of the regulation error are zero.

The cascade structure of (2) with (4) suggests to use forwarding techniques, see, e.g., [24, 25]. We approach the problem by 
considering the nominal plant (2) with $w=0$ and we show how the plant stabilizer introduced in Assumption 1 can be completed to include also the (critically stable) internal model unit.

To this end, we first introduce the following function

$$
\mathcal{M}(x):=\lim _{t \rightarrow \infty} \int_{0}^{t} \exp (\Phi s) \Gamma h\left(\varphi_{x}(x, s), 0\right) d s
$$

in which $\varphi_{x}(x, s)$ is the trajectory of $\dot{x}=f(x, 0)+g(x, 0) \alpha(x)$ at time $s$ with initial condition $x$ at time $s=0$. The following result holds.

Lemma 1 Under Assumption 1, the function $\mathcal{M}: \mathcal{A} \rightarrow \mathbb{R}^{v p}$ defined in (7) is $C^{2}$ and solution of

$$
\frac{\partial \mathcal{M}}{\partial x}(x)(f(x, 0)+g(x, 0) \alpha(x))=\Phi \mathcal{M}(x)+\Gamma h(x, 0) .
$$

Moreover, if Assumption 2 holds, then the pair $\left(B^{\top} M^{\top}, \Phi\right)$ is observable, where $B$ is defined in (3) and

$$
M:=\frac{\partial \mathcal{M}}{\partial x}(0)
$$

Proof: The fact that (7) is a solution to (8) can be established by following [24, Lemma IV.2]. Then, in order to show the observability of the pair $\left(B^{\top} M^{\top}\right)$, linearize the PDE (7) around the origin. We obtain

$$
M(A+B N)=\Phi M+\Gamma C
$$

where $N=\frac{\partial \alpha}{\partial x}(0)$. In light of Assumption 1, the matrix $A+B N$ is Hurwitz. Recall that the matrix $\Phi$ is neutrally stable. Therefore, the solution of the previous Sylvester equation is unique since the spectra of $(A+B N)$ and $\Phi$ are disjoint.

Then, let $-\lambda$ be an eigenvalue of $\Phi$ and let $v$ be its associated eigenvector, i.e. $-\lambda v=\Phi v$. Since $\Phi$ is skew-symmetric also $\lambda$ is an eigenvalue of $\Phi$. Furthermore, $\Phi=-\Phi^{\top}$. As a consequence

$$
(-\lambda v)^{\top}=(\Phi v)^{\top}=v^{\top} \Phi^{\top}=-v^{\top} \Phi \quad \Longrightarrow \quad \lambda v^{\top}=v^{\top} \Phi .
$$

By pre-multiplyig equation (20) by $v^{\top}$,

$$
v^{\top} M(A+B N)=v^{\top} \Phi M+v^{\top} \Gamma C
$$

which implies

$$
v^{\top} M(\lambda I-A-B N)+v^{\top} \Gamma C=0 .
$$

Let assume that $v$ is the in kernel of $M^{\top} B^{\top}$, namely $B^{\top} M^{\top} v=0$ and therefore $v^{\top} M B=0$. By collecting the previous relations, and by using the fact that $v^{\top} M B N=0$, we get

$$
\left(\begin{array}{ll}
v^{\top} M & v^{\top} \Gamma
\end{array}\right)\left(\begin{array}{cc}
\lambda I-A & B \\
C & 0
\end{array}\right)=0 .
$$

But this contradicts the Assumption 4. As a consequence there is no non-zero vector $v$ satisfying

$$
\left(\begin{array}{c}
\lambda I-\Phi \\
B^{\top} M^{\top}
\end{array}\right) v=0
$$

and therefore the PBH observability test

$$
\operatorname{rank}\left[\begin{array}{c}
\lambda I-\Phi \\
B^{\top} M^{\top}
\end{array}\right]=n \quad \forall \lambda \in \sigma(\Phi)
$$

where $\sigma(\Phi)$ denotes the spectrum of $\Phi$, is satisfied, concluding the proof.

The function $\mathcal{M}$ is the seed to design a state-feedback stabilizer of the form (6) for the system (2), (4) with $w=0$. For this, recall that, in view of Assumption 1, a converse Lyapunov function (see, for instance, [29]) can be used to establish the existence of a $C^{1}$ function $V: \mathcal{A} \rightarrow \mathbb{R}$ which is positive definite and proper on $\mathcal{A}$ and a positive definite function $W: \mathcal{A} \rightarrow \mathbb{R}$ quadratic around the origin such that

$$
\frac{\partial V}{\partial x}(x) f(x, 0) \leq-W(x) \quad \forall x \in \mathcal{A} .
$$

Then, let $\theta: \mathcal{A} \times \mathbb{R}^{v} \rightarrow \mathbb{R}^{m}$

$$
\theta(x, \xi):=-b\left(\frac{\partial V(x)}{\partial x} g(x, 0)\right)^{\top}+\left(\frac{\partial \mathcal{M}(x)}{\partial x} g(x, 0)\right)^{\top} \Lambda(\xi-\mathcal{M}(x))
$$

where $b, \Lambda$ are degree-of-freedom that can be used to tune the performances of the control law, with $b>0$ and $\Lambda>0$ being any matrix satisfying $\Lambda \Phi+\Phi^{\top} \Lambda=0$. The following result then holds.

Theorem 1 Let Assumptions 1 and 2 hold. Then, the origin of the system (2), (4) with $w=0$ controlled by (6) with

$$
K(x, \xi)=\alpha(x)+\theta(x, \xi)
$$

where $\alpha$ is given by Assumption 1 and $\theta$ is selected as (10), is asymptotically and locally exponentially stable with $\mathcal{A} \times \mathbb{R}^{v p}$ as domain of attraction.

Proof: For compactness, in the rest of this proof we will denote $f(x):=f(x, 0), g(x):=g(x, 0), h(x):=h(x, 0)$. Consider the function $U: \mathbb{R}^{n} \times \mathbb{R}^{v p} \rightarrow \mathbb{R}$ defined as

$$
U(x, \xi):=b V(x)+\frac{1}{2}(\xi-\mathcal{M}(x))^{\top} \Lambda(\xi-\mathcal{M}(x)) .
$$

In view of the properties of $V$ and $\mathcal{M}$, such a function $U$ satisfies

$$
\underline{a}\left(|x|\left[1+\frac{1}{d(x, \partial \mathcal{A})}\right]+|\xi|\right) \leq U(x, \xi), \quad \forall x \in \mathcal{A},
$$

for some class- $\mathcal{K}_{\infty}$ function $\underline{a}$ which is quadratic near the origin, and with $d(x, \partial \mathcal{A})$ denoting the distance of $x$ from the set boundary of the closure of the set $\mathcal{A}$, see for instance [9, Appendix A]. Moreover, $U$ is proper on $\mathcal{A} \times \mathbb{R}^{v p}$. Now, by deriving $U$, and by using (8), (9), and by recalling that $\Lambda \Phi+\Phi^{\top} \Lambda=0$, the following holds

$$
\begin{aligned}
\dot{U} \leq & b \frac{\partial V}{\partial x}(f(x)+g(x)(\alpha(x)+\theta(x, \xi))) \\
& +(\xi-\mathcal{M}(x))^{\top} \Lambda\left[\Phi \xi+\Gamma h(x)-\frac{\partial \mathcal{M}}{\partial x} f(x)\right. \\
& \left.\quad-\frac{\partial \mathcal{M}}{\partial x} g(x)(\alpha(x)+\theta(x, \xi))\right] \\
\leq & -b W(x)+\left(b \frac{\partial V}{\partial x}-(\xi-\mathcal{M}(x))^{\top} \Lambda \frac{\partial \mathcal{M}}{\partial x}\right) g(x) \theta(x, \xi) \\
\leq & -b W(x)-\theta(x, \xi)^{\top} \theta(x, \xi) .
\end{aligned}
$$


By La Salle arguments the solution then converges to the largest invariant set $\mathcal{I}$ contained in the set

$$
\left\{(x, \xi) \in \mathbb{R}^{n} \times \mathbb{R}^{v p}: W(x)=0, \theta(x, \xi)=0\right\} .
$$

By using the fact that $W(x)=0$ if and only if $x=0$, that $\frac{\partial V}{\partial x}(0)=0$ and that $\mathcal{M}(0)=0$, the previous set reduces to

$$
\left\{(x, \xi) \in \mathbb{R}^{n} \times \mathbb{R}^{v p}: x=0, B^{\top} M^{\top} \Lambda \xi=0\right\},
$$

with $B$ defined in (3) and $M$ defined in Lemma 1 . Hence, by using the fact that the pair $\left(B^{\top} M^{\top}, \Phi\right)$ is observable (see Lemma 1 ), we conclude that the set $\mathcal{I}$ is the origin. Therefore the origin is asymptotically stable with a domain of attraction $\mathcal{A} \times \mathbb{R}^{v p}$. Locally exponentially stability immediately follows from Assumption 1 from linearization of the closed-loop system at the origin.

It is worth observing that the design of $\theta$ in (10) relies on the exact knowledge of the function $V, \mathcal{M}$, but alternative designs of a stabilized feedback law based on the approximation of $V$ and/or $\mathcal{M}$ are possible, see, for instance [24], [25, Section III] and references therein.

The proposed state feedback stabilizer succeeds in making the origin of the nominal system asymptotically and locally exponentially stable when $w=0$ by preserving the domain of attraction enforced by the stabilizer of the regulated plant and globally with respect of the initial state of the internal model unit. The next theorem, which is the main result of the paper, shows the property of the stabilizer when applied to the real process (1) and when the exosystem signal $w(t)$ is injected in the loop. It is shown that the trajectory of the system originated by an arbitrary large compact set contained in $\mathcal{A} \times \mathbb{R}^{v p}$ are asymptotically attracted by a $T$-periodic trajectory provided that the real process is "sufficiently" close to the nominal plant and the amplitude of the exosystem is sufficiently small. Closeness of the real process to the nominal model is expressed in terms of the following functions

$$
\begin{aligned}
\Delta_{f}(w, x, \xi) & :=\tilde{f}(w, x, K)-(f(w, x)+g(w, x) K) \\
\Delta_{h}(w, x, \xi) & :=\tilde{h}(w, x, K)-h(w, x) \\
\Delta_{\partial_{x} f}(w, x, \xi) & :=\frac{\partial \tilde{f}}{\partial x}(w, x, K)-\left(\frac{\partial f}{\partial x}(w, x)+\frac{\partial g}{\partial x}(w, x) K\right) \\
\Delta_{\partial_{u} f}(w, x, \xi) & :=\frac{\partial \tilde{f}}{\partial u}(w, x, K)-g(w, x) K \\
\Delta_{\partial_{x} h}(w, x, \xi) & :=\frac{\partial \tilde{h}}{\partial x}(w, x, K)-\frac{\partial h}{\partial x}(w, x) \\
\Delta_{\partial_{u} h}(w, x, \xi) & :=\frac{\partial \tilde{h}}{\partial u}(w, x, K)
\end{aligned}
$$

in which, for easy of notation, we set $K=K(x, \xi)$.

Theorem 2 Let Assumptions 1 and 2 hold and let $K(x \xi)$ be fixed as in Theorem 1. Let $X \times \Xi \subset \mathcal{A} \times \mathbb{R}^{v p}$ be an arbitrary compact set and let $\mathcal{S} \subset \mathcal{A} \times \mathbb{R}^{v p}$ be the forward invariant set containing the trajectories of (2), (4) with $w=0$ originating from initial conditions in $X \times \Xi \subset \mathcal{S}$. Then, for all compact sets $X^{\prime} \subset X$ and $\Xi^{\prime} \subset \Xi$ there exist positive $\delta$ and $\bar{w}$ such that for any $C^{1} T$-periodic trajectory satisfying $\|w(t)\| \leq \bar{w}$ for all $t \geq 0$ and any real process satisfying

$$
\begin{gathered}
\left\|\Delta_{f}(w, x, \xi)+\Delta_{h}(w, x, \xi)\right\| \leq \delta \\
\left\|\left(\begin{array}{cc}
\Delta_{\partial_{x} f}(w, x, \xi) & \Delta_{\partial_{u} f}(w, x, \xi) \\
\Delta_{\partial_{x} h}(w, x, \xi) & \Delta_{\partial_{u} h}(w, x, \xi)
\end{array}\right)\right\| \leq \delta
\end{gathered}
$$

for all $(x, \xi) \in \mathcal{S}$ and $\|w\| \leq \bar{w}$, the actual closed-loop system (1), (4), (6) has a $C^{1} T$-periodic solution $\left(x^{\circ}(t), \xi^{\circ}(t)\right)$ which is asymptotically stable with a domain of attraction containing $X^{\prime} \times \Xi^{\prime}$. As a consequence, the Fourier coefficients of the regulation error $e(t)$ associated to the frequencies $2 k \pi / T$, with $k=0,1, \ldots, v$, are asymptotically vanishing.

Proof: Consider the closed-loop system (2), (4), (6) with $w=0$. Following the same line of [25, Proposition 3], direct application of [25, Lemma 5] establishes, for some sufficiently small $\delta>0$, the existence of an equilibrium $x^{\circ}, \xi^{\circ}$ (possibly different from the origin) which is locally exponentially stable and asymptotically stable with a domain of attraction containing $\mathcal{S}$, for the closed-loop system (1), (4), (6). Rewriting the system closed-loop system (1), (4), (6) in the error coordinates $x-x^{\circ}$ and $\xi^{\circ}$ allows to apply Theorem 3 given in the Appendix to show the existence of a periodic solution which is asymptotically stable with a domain of attraction including $X^{\prime} \times \Xi^{\prime}$. Applying Proposition 1 on such steady-state periodic solution, the proof concludes.

Remark 1 The control law proposed in Theorem 1 and 2 is based on the full knowledge of the state $x$. When $x$ is not fully available, an output-feedback approach can be pursued by means of state-observers. We refer to [25] for further details. $\lrcorner$

We remark that the proof of Theorem 2 is based on a (conservative) "total-stability" result that involves the stability margin of the closed-loop system. Such a stability margin may in general decrease with the number of oscillators. As a consequence, the admissible size $\bar{w}$ of the exosignals $w$ may in principle decrease to zero by letting the number of oscillators $v$ go to infinity. However, as shown in the technical result [30] for the particular case of minimum-phase systems, this is not the case. In other words, one can chose an arbitrary number of oscillators, by preserving the same properties with respect to the size $\bar{w}$ of the exosignal $w$, and at the same by improving the $L_{2}$ norm of the asymptotic output error $e$, namely improving the approximate output regulation objective in the following sense

$$
\lim _{v \rightarrow \infty} \limsup _{t \rightarrow \infty} e(t)=0 .
$$

\section{New insights on linear output regulation}

In this section we revise the design procedure proposed in section 2 for linear systems of the form

$$
\begin{aligned}
\dot{x} & =A x+B u+P w \\
e & =C x+Q w .
\end{aligned}
$$


In this linear context, we follow the standard paradigm of output regulation $[1,2]$ by supposing that that $w$ is generated by an autonomous system of the form

$$
\dot{w}=S w
$$

with $w \in \mathbb{R}^{\rho}$. In this context, the Assumptions 1 and 2 read as follow. Recall that they are necessary and sufficient for the existence of a robust regulator.

Assumption 3 (Stabilizability) The pair $(A, B)$ is stabilizable.

Assumption 4 (Non-resonance condition) The following matrix

$$
\left(\begin{array}{cc}
A-\lambda I & B \\
C & 0
\end{array}\right)
$$

has independent rows for each $\lambda$ which is an eigenvalue of $S$.

The internal model unit is simply designed by adding $p$ copies of the exosystem dynamics on the regulation error as in (4), where $(\phi, G)$ is a controllable pair with $\phi$ such that it's characteristic polynomial coincides with the minimal polynomial of $S$. The control law (6) is then selected in this context as

$$
u=K_{x} x+K_{\xi} \xi
$$

making the resulting closed loop system asymptotically stable when $w=0$, namely so that the matrix

$$
A_{\mathrm{cl}}:=\left(\begin{array}{cc}
A+B K_{x} & B K_{\xi} \\
\Gamma C & \Phi
\end{array}\right)
$$

is Hurwitz. The regulator given by (4), (14) solves the asymptotic output regulation problem. As a matter of fact, by letting the closed-loop dynamics

$$
\begin{aligned}
\dot{w} & =S w \\
\dot{x}_{e} & =A_{\mathrm{cl}} x_{e}+P_{\mathrm{cl}} w
\end{aligned}
$$

in which $x_{e}:=\operatorname{col}(x, \xi)$ and $P_{\mathrm{cl}}$ is an appropriately defined matrix, the fact that $A_{\mathrm{cl}}$ is Hurwitz guarantees that $x_{e}(t)$ asymptotically reaches a steady-state of the form $\Pi_{e} w(t)$ with $\Pi_{e}$ solution of the Sylvester equation

$$
\Pi_{e} S-A_{\mathrm{cl}} \Pi_{e}=P_{\mathrm{cl}} .
$$

Furthermore, by partitioning $\Pi_{e}$ as $\Pi_{e}=\operatorname{col}\left(\Pi_{x}, \Pi_{\xi}\right)$ coherently with the definition of $x_{e}$, the fact that the characteristic polynomial of $\phi$ coincides with the minimal polynomial of $S$ can be used to prove that

$$
C \Pi_{x}+Q=0,
$$

namely the regulation error converges to zero asymptotically. Note that this property is robust to small parametric uncertainties affecting the matrices in $(12)$, see $[1,2]$. In addition, it is immediately realized that the matrices $\left(\Pi_{x}, \Pi_{\xi}\right)$, whose existence is guaranteed by $A_{\mathrm{cl}}$ Hurwitz, fulfill the relation

$$
\Pi_{x} S=A \Pi_{x}+B \Psi+P
$$

with $\Psi:=K_{x} \Pi_{x}+K_{\xi} \Pi_{\xi}$. The set of equations (17)-(18), interpreted as equations in the unknowns $\Pi_{x}$ and $\Psi$, are recognized to be the "regulator equations" linked to (12) and (13), expressing the desired steady-state for the state $x(t)$, which is $\Pi_{x} w(t)$, and for the input $u(t)$, which is $\Psi w(t)$. Stabilizability of the extended system (12), (4) with $w=0$, namely Assumptions 3 and 4 , are thus sufficient conditions to solve the regulator equations (17)-(18) for any instance of the pair $(P, Q)$. Related to this, it's a well known fact (see, e.g., [31, Lemma 1.4,2]) that (17)-(18) admit a solution $\left(\Pi_{x}, \Psi\right)$ for all possible set of matrices $(P, Q)$ if and only if the non resonance condition expressed by Assumption 4 holds.

Now, the tools presented in Section 2 can be clearly specialized to the case of linear systems (12) by obtaining a possible design strategy for the linear stabilizer (14), namely of the matrices $K_{x}$ and $K_{\xi}$. The strategy can be summarized in a few steps as follows. By using Assumption 3, let $N$ be a matrix such that $A+B N$ is Hurwitz and let $\mathcal{P}=\mathcal{P}^{\top}>0$ be the solution of the Lyapunov equation

$$
\mathcal{P}(A+B N)+(A+B N)^{\top} \mathcal{P}=-2 a I
$$

with $a>0$. Then, let $M$ be the solution of the following Sylvester equation (see (8))

$$
M(A+B N)=\Phi M+\Gamma C .
$$

Since the spectra of $(A+B N)$ and $\Phi$ are disjoints, such a solution is well defined and unique. Then, with an eye to (10), (11), select the matrices $K_{x}$ and $K_{\xi}$ of (14) as

$$
K_{x}=N-b B^{\top} \mathcal{P}-B^{\top} M^{\top} \Lambda M, \quad K_{\xi}=B^{\top} M^{\top} \Lambda,
$$

where $b>0$ and $\Lambda>0$ are degree-of-freedom with $\Lambda$ satisfying $\Lambda \Phi+\Phi^{\top} \Lambda=0$. If Assumption 4 holds, then the closed-loop system matrix (15) is Hurwitz (see Theorem 1 specialized to the linear case), and the output regulation problem is solved.

The next proposition establishes a deep connection between the non-resonance condition of Assumption 4, the existence of a solution to the regulator equations (18), (17), and the observability property of the pair $\left(B^{T} M^{T}, \Phi\right)$, showing that these conditions are indeed equivalent.

Proposition 2 Consider system (12) with exosystem (13). Suppose $S$ is neutrally stable, and Assumptions 3 hold. Then, the following sentences are equivalent.

(i) There exist matrices $\Pi_{x}$, $\Psi$ solution of the regulator equations (17), (18) for any matrices $P, Q$.

(ii) Assumption 4 holds.

(iii) Let $\Phi, \Gamma$ be selected as in (4), let $N$ be any matrix such that $\sigma(A+B N) \cap \sigma(\Phi)=\emptyset$ and let $M$ be solution of (20). The pair $\left(B^{\top} M^{\top}, \Phi\right)$ is observable.

Proof: The implications $(i) \Leftrightarrow$ (ii) are given in [31, Lemma 1.4.2]. Therefore, we will prove only the implications $(i) \Leftrightarrow$ (iii). 
First, we prove that (iii) $\Rightarrow($ i). To this end, select $u$ as in (14) with $K_{x}=N-B^{\top} M^{\top} M^{\top} M$ and $K_{\xi}=B^{\top} M^{\top}$. Applying the linear change of coordinates $\xi \mapsto \eta:=\xi-M x$ the closed-loop system (15) reads

$$
\begin{aligned}
\dot{w} & =S w \\
\dot{\tilde{x}}_{e} & =\tilde{A}_{\mathrm{cl}} \tilde{x}_{e}+\tilde{P}_{\mathrm{cl}} w
\end{aligned}
$$

with $\tilde{x}_{e}=\operatorname{col}(x, \eta)$ and

$$
\tilde{A}_{\mathrm{cl}}:=\left(\begin{array}{cc}
A+B N & B B^{\top} M \\
0 & \Phi-M B B^{\top} M
\end{array}\right)
$$

and some appropriate defined matrix $\tilde{P}$. Since the matrix $\Phi$ is neutrally stable and the pair $\left(B^{\top} M^{\top}, \Phi\right)$ is observable, the matrix $\Phi-M B B^{\top} M^{\top}$ is Hurwitz (this can be shown by using LaSalle like arguments). Hence, due to the block-triangular structure, the fact that the spectrum of $A+B N$ and $S$ are disjoint, and $\Phi-M B B^{\top} M^{\top}$ is Hurwitz, we conclude that the spectrum of $\tilde{A}_{\mathrm{cl}}$ and $S$ are disjoint. Since the matrix $\tilde{A}_{\mathrm{cl}}$ and $A_{\mathrm{cl}}$ are similar, for any $(P, Q)$ the Sylvester equation (16) admits a unique solution. Controllability of the pair $(\Phi, \Gamma)$ implies (17) and with $\Psi=K_{x} \Pi_{x}+K_{\xi} \Pi_{\xi}$ we obtain (18) concluding the first part of the proof.

We prove now that $(i) \Rightarrow$ (iii) by contradiction. In particular, assume a solution to the regulator equations (17), (18) exists. Let $N$ be any matrix such that the spectra of $\Phi$ and $(A+B N)$ are disjoints. This is always possible by Assumption 3 and the fact that $\Phi$ is neutrally stable. Let the pair $(\Phi, \Gamma)$ controllable and $M$ solution to (20). Now, by adding and subtracting the term $B N \Pi_{x}$, and by pre-multiplying by $M$ equation (18) we get

$$
\begin{aligned}
M \Pi_{x} S & =M(A+B N) \Pi_{x}+M B\left(\Psi-N \Pi_{x}\right)+M P \\
0 & =C \Pi_{x}+Q .
\end{aligned}
$$

By using (20), we further obtain

$$
\begin{aligned}
M \Pi_{x} S & =(\Phi M+\Gamma C) \Pi_{x}+M B\left(\Psi-N \Pi_{x}\right)+M P \\
0 & =C \Pi_{x}+Q,
\end{aligned}
$$

and therefore, by multiplying the second equation by $\Gamma$, it yields

$$
M \Pi_{x} S=\Phi M \Pi_{x}+\left(M P-\Gamma Q+M B\left(\Psi-N \Pi_{x}\right)\right) .
$$

Now let $-\lambda$ be an eigenvector of $\Phi$ and suppose the pair $\left(B^{\top} M^{\top}, \Phi\right)$ is not observable, namely there exists $v$ satisfying

$$
\Phi v=-\lambda v, \quad B^{\top} M^{\top} v=0 .
$$

By using skew symmetry of $\Phi$, it follows that $v$ also satisfies $v^{\top} \Phi=\lambda v^{\top}$. Since $\Phi$ and $S$ have the same spectrum, there exists a $w \neq 0$ satisfying $S w=\lambda w$. As a consequence, by premultiplying (22) by $v^{\top}$ and by post-multiplying by $w$ we get

$$
\begin{aligned}
v^{\top} M \Pi_{x} S w=v^{\top} \Phi M \Pi_{x} w & \\
& +v^{\top}\left(M P-\Gamma Q+M B\left(\Psi-N \Pi_{x}\right)\right) w
\end{aligned}
$$

and

$$
\begin{aligned}
v^{\top}\left(M \Pi_{x}\right) \lambda w=\lambda v^{\top}\left(M \Pi_{x}\right) w & \\
& +v^{\top}\left(M P-\Gamma Q+M B\left(\Psi-N \Pi_{x}\right)\right) w .
\end{aligned}
$$

and $v^{\top}(M P-\Gamma Q) w=0$. The latter can be expressed as

$$
\sum_{j, k, \ell} v_{j} M_{j, k} P_{k, \ell} w_{\ell}-\sum_{j, k, \ell} v_{j} \Gamma_{j, k} Q_{k, \ell} w_{\ell}=0 .
$$

By differentiating the previous equality with respect to $P_{\ell, \ell}$ we obtain

$$
\begin{aligned}
\frac{\partial}{\partial P_{\ell, \ell}}\left(\sum_{j, k, \ell} v_{j} M_{j, k} P_{k, \ell} w_{\ell}-\sum_{j, k, \ell} v_{j} \Gamma_{j, k} Q_{k, \ell} w_{\ell}\right) & = \\
\sum_{j, k, \ell} v_{j} M_{j, k} w_{\ell} & =0,
\end{aligned}
$$

for all $k, \ell$. Similarly, by differentiating with respect to $Q_{k, \ell}$, we obtain

$$
\begin{aligned}
\frac{\partial}{\partial Q_{k, \ell}}\left(\sum_{j, k, \ell} v_{j} M_{j, k} P_{k, \ell} w_{\ell}-\sum_{j, k, \ell} v_{j} \Gamma_{j, k} Q_{k, \ell} w_{\ell}\right) & = \\
\sum_{j, k, \ell} v_{j} \Gamma_{j, k} w_{\ell} & =0,
\end{aligned}
$$

for all $k, \ell$. Now let $\ell$ be such that $w_{\ell} \neq 0$. From the previous expressions we get $v^{\top} M=0$ and $v^{\top} \Gamma=0$. By using the fact that $v^{\top} \Phi=\lambda v^{\top}$ we have

$$
\begin{aligned}
v^{\top}\left[\begin{array}{llll}
\Gamma & \Phi \Gamma & \cdots & \Phi^{(r \times p)-1} \Gamma
\end{array}\right] \\
=v^{\top}\left[\begin{array}{llll}
\Gamma & \lambda \Gamma & \cdots & \lambda^{(r \times p)-1} \Gamma
\end{array}\right]=0,
\end{aligned}
$$

which contradicts the fact that the pair $(\Phi, \Gamma)$ is controllable. Hence, the pair $\left(B^{\top} M^{\top}, \Phi\right)$ must be observable and this concludes the proof.

The novelty and the interest of Proposition 2 is that, under the stabilizability Assumption 3, we can ensure the existence of a regulator solving the output regulation problem for linear systems, with a new set of necessary and sufficient conditions, expressed by the item (iii), which can be checked by means of simple and standard computational tools, namely the resolution of a Sylvester equation. Although this result may not be always useful in the finite-dimensional linear context ${ }^{1}$, such a result may be of large interest for other classes of systems for which the computations of the invariant zeros is not an easy task. For instance, in the context of infinite dimensional systems, the computation of the range of an operator (i.e. the equivalent condition in terms of the rank of a matrix) or the characterization of its spectral properties are not always easy (see, e.g., [33, Assumption 2.2]). In this case, checking the observability property may result to be an easier task. This is what happens, for instance, in the context of the use of an integral action via forwarding feedback for open-loop exponentially stable PDEs, see, e.g. [34, Section III].

\section{Bilinear Systems}

As a special case of the system (2), consider the class of single-input single-output bilinear systems that can be written

\footnotetext{
${ }^{1}$ As a matter of fact, algorithms for the computation of invariant zeros of a linear system are well known, see, e.g. [32].
} 
in the form

$$
\begin{aligned}
\dot{x} & =A x+(D x+B) u+P w \\
e & =C x+Q w
\end{aligned}
$$

where $x \in \mathbb{R}^{n}, u, e \in \mathbb{R}$, and $A, B, C, D, R, Q$ are matrices of appropriate dimensions. Note that, generically, bilinear systems have not a well-defined relative degree since the term $D x+B$ may posses some singularities and vary its rank. Hence, we cannot put the system in the canonical normal form employed in standard output regulation problems such as [8,9]. In this section, we follow the recipe given in Section 2.

For the sake of simplicity, we suppose that the matrix $A$ is Hurwitz. With respect to Assumption 1, we have $\alpha(x)=0$. It is worth recalling that numerous control engineering applications satisfy such an "open-loop stability" assumption, see for instance the case of heat-exchangers [26] or power converters [27]. We define $\mathcal{P}$ as the solution to the Lyapunov matrix inequality

$$
\mathcal{P} A+\mathcal{P} A^{\top} \leq-2 a I
$$

so that the inequality (9) is satisfied with $V=x^{\top} \mathcal{P} x$ and $W=-2 a|x|^{2}$. Then, we suppose that the triplet $(A, B, C)$ satisfies Assumption 2. We design a regulator of the form (4), (5). Therefore, the function $\mathcal{M}$ in Lemma 1 is a linear function obtained as solution to the Sylvester equation

$$
M A=\Phi M+\Gamma C
$$

and the state-feedback law (10) reads

$$
\theta(x, \xi)=-2 b(D x+B)^{\top} \mathcal{P} x+(D x+B)^{\top} M^{\top} \Lambda(\xi-M x) .
$$

As a numerical example, we consider system (23) with nominal parameters $A, B, C, D$ given by

$$
A=\left(\begin{array}{cc}
-1 & 1 \\
0 & -2
\end{array}\right), B=\left(\begin{array}{l}
1 \\
1
\end{array}\right), D=\left(\begin{array}{cc}
0.1 & 0.2 \\
-0.2 & 0.3
\end{array}\right), C=\left(\begin{array}{ll}
1 & 0
\end{array}\right)
$$

and $P, Q$ any non-zero matrices of unitary norm. We finally consider the internal-model based regulator (4), (5), (24). In the simulations, we consider $w(t)=w_{0}+w_{1} \sin (2 \pi t)$ with $\left|w_{0}\right| \leq 1$, $\left|w_{1}\right| \leq 1$. In particular $w_{0}=0.5$ and $w_{1}=\sqrt{2} / 2$. In simulations we considered different scenarios by varying the number of oscillators $v$ from 1 to 6 . In Table 1, we reported the asymptotic value of the regulated output $e$, confirming the fact that, when augmenting the number of oscillators, the approximated regulation objective is improved. Figure 1 shows transient behaviors with initial conditions $x(0)=(10,-7), \xi(0)=0$, and with the number of oscillators $v$ selected as $v=6$. Note that, in order to improve the transient behavior (which is very slow due to the presence of the oscillators), one may use the technique proposed in [35].

The obtained simulations confirm the preliminary results in [20] for the special class of bilinear systems, and show similar results to those obtained for the class of minimum-phase systems in [30].

\begin{tabular}{cc}
\hline$v$ & $\limsup _{t \rightarrow \infty}|e(t)|$ \\
\hline 1 & $10^{-2}$ \\
2 & $9.9 \cdot 10^{-4}$ \\
3 & $1.2 \cdot 10^{-4}$ \\
4 & $2.2 \cdot 10^{-5}$ \\
5 & $1.3 \cdot 10^{-5}$ \\
\hline
\end{tabular}

Table 1: Asymptotic value of the regulated output $e$ in (23) depending on the number of oscillator $v$.

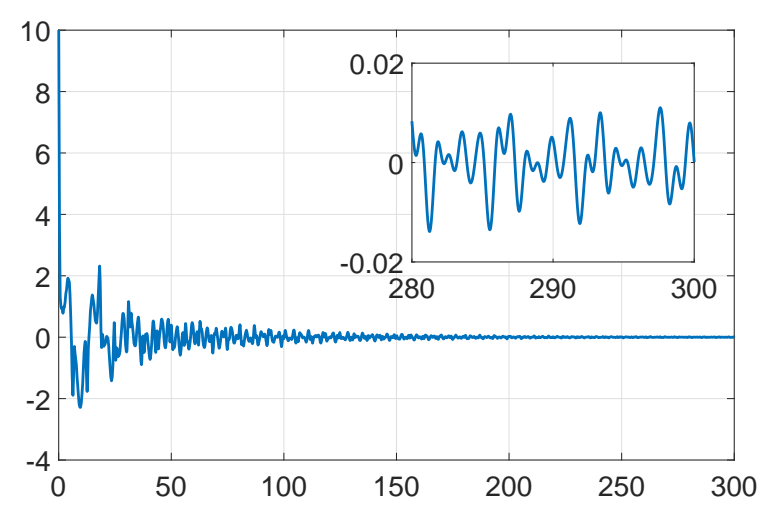

Figure 1: Behavior of the regulated output of system (23)-(24) with an internal model unit compose of 6 oscillators.

\section{Conclusions}

We investigated the problem of output regulation for multiinput multi-output input-affine nonlinear systems in presence of periodic exogenous trajectories. Following the linear paradigm, we proposed an internal model approach which lies on the design principle "add harmonics on the regulation error and stabilize the extended system". A simple state-feedback design based on forwarding approach is then proposed. The asymptotic behavior of the closed-loop trajectories in presence of arbitrarily small perturbations of the plant's model is analyzed and it is shown that harmonic regulation is obtained, namely the Fourier coefficients linked to the frequencies copied in the internal model are canceled on the asymptotic error. The stability properties of the closed-loop system are semi-global in the size of the initial conditions of the plant but only local in the size of the perturbation, i.e., its magnitude has to be small enough. A dependence between the size of the exosignals and the number of oscillator is not completely clear. However, the technical results $[36,30]$ developed in in the context of minimum-phase systems suggest that this is not always the case, namely augmenting the number of oscillators has no influence on the admissible size of the perturbation and/or references. We conjecture that in order to achieve global results, incremental stability properties may need to be ensured. Preliminary results in this direction for the simple case of integral action have been investigated in [37].

Finally, as a by-product of the proposed forwarding approach, we shed light on the linear case by presenting a new necessary and sufficient condition under which the linear ro- 
bust output regulation problem can be solved. The proposed condition doesn't rely on the so-called non-resonance assumption and it is easy to check from the computational point of view.

\section{AppendixA. Existence of stable periodic solutions}

The following result concerns the existence and stability of a periodic solution to nonlinear system forced by a periodic input.

Theorem 3 Let be given a $C^{1}$ function $\varphi: \mathbb{R}^{n} \times \mathbb{R}^{\rho} \rightarrow \mathbb{R}^{n}$ such that the origin of

$$
\dot{x}=\varphi(x, 0)
$$

is asymptotically, locally exponentially, stable with a domain of attraction $\mathcal{A} \subseteq \mathbb{R}^{n}$. Then, for any compact set $X \subset \mathcal{A}$, there exists $\varepsilon>0$ such that, for any $C^{1} T$-periodic function $w: \mathbb{R} \rightarrow \mathbb{R}^{\rho}$ satisfying $\sup _{s \in[0, T]}|w(s)| \leq \varepsilon$, the system

$$
\dot{x}=\varphi(x, w)
$$

admits a unique $T$-periodic solution $x^{\circ}$ which is asymptotically stable with a domain of attraction that includes $X$.

Proof: Although we could not find in literature any reference containing the exact statement of Theorem 3, we stress that such a theorem is a direct consequence of well known results on the existence and stability of periodic solutions (see, e.g., [38], [39], [40] or [41, Chapter 4]). We just sketch its proof for completeness.

First, by using the fact that the origin of (A.1) is asymptotically stable, it can be shown that for any compact set $X \subset \mathcal{A}$, there exists $\varepsilon^{\star}>0$, and for any $\varepsilon \in\left[0, \varepsilon^{\star}\right)$ there exists a positive real number $\bar{x}(\varepsilon) \geq 0$, such that solutions to system (A.2) starting from $X$, with $w$ satisfying $|w(t)| \leq \varepsilon$ for all $t \geq 0$, are complete forward in time and moreover $\lim _{t \rightarrow \infty}|x(t)| \leq \bar{x}(\varepsilon)$. Furthermore such an ultimate bound satisfies $\lim _{\varepsilon \rightarrow 0} \bar{x}(\varepsilon)=0$. This can be established, for instance, by combining the arguments of [42, Theorem 10.2.1] and [9, Theorem 4 and Lemma 1]. The main idea consists in using a converse Lyapunov theorem for system (A.1) and selecting the value of $\varepsilon^{\star}>0$ small enough according to the Lipschitz constant of $\varphi(x, w)-\varphi(x, 0)$ on the set $X$.

Next, we can show that there exists $r>0$ small enough such that, for any $C^{1} T$-periodic signal $w$ satisfying $\sup _{s \in[0, T]}|w(s)| \leq$ $r$, system (A.2) admits a unique $C^{1} T$-periodic solution satisfying $\sup _{s \in[0, T]}|x(s)| \leq r$ which is asymptotically stable with a domain of attraction which includes the set $\left\{x \in \mathbb{R}^{n}:|x| \leq 2 r\right\}$. This can be shown by rewriting (A.2) as

$$
\dot{x}=A x+\Delta(x, w), \quad \Delta(x, w):=\varphi(x, w)-A x,
$$

where the matrix $A:=\frac{\partial f}{\partial x}(0,0)$ is Hurwitz due to the locally exponential stability properties of system (A.1), and using standard arguments in differential equations analysis such as in [40, $\S 8$, Theorem 5.1, Theorem 6.2]. The main idea consists in constructing the periodic solution by using the constant variation formula and applying Banach fixed point theorem to show that, thanks to the exponential stability, the obtained operator is a contraction. Finally, selecting $\varepsilon$ small enough so that $\bar{x}(\varepsilon)<2 r$, ensures the statement of the theorem.

\section{References}

[1] B. A. Francis, W. M. Wonham, The internal model principle of control theory, Automatica 12 (5) (1976) 457-465.

[2] E. Davison, The robust control of a servomechanism problem for linear time-invariant multivariable systems, IEEE transactions on Automatic Control 21 (1) (1976) 25-34.

[3] A. Isidori, C. I. Byrnes, Output regulation of nonlinear systems, IEEE transactions on Automatic Control 35 (2) (1990) 131-140.

[4] C. I. Byrnes, A. Isidori, Limit sets, zero dynamics, and internal models in the problem of nonlinear output regulation, IEEE Transactions on Automatic Control 48 (10) (2003) 1712-1723.

[5] F. D. Priscoli, Robust tracking for a class of nonlinear plants, achieved via a linear controller, in: Proceedings of 32nd IEEE Conference on Decision and Control, 3550-3555, 1993.

[6] M. Bin, L. Marconi, The chicken-egg dilemma and the robustness issue in nonlinear output regulation with a look towards adaptation and universal approximators, in: 57th IEEE Conference on Decision and Control (CDC), 5391-5396, 2018.

[7] L. Wang, L. Marconi, C. Wen, H. Su, Pre-processing nonlinear output regulation with non-vanishing measurements, Automatica 111 (2020) 108616.

[8] C. I. Byrnes, A. Isidori, Nonlinear internal models for output regulation, IEEE Transactions on Automatic Control 49 (12) (2004) 2244-2247.

[9] L. Marconi, L. Praly, A. Isidori, Output stabilization via nonlinear Luenberger observers, SIAM Journal on Control and Optimization 45 (6) (2007) 2277-2298.

[10] F. Forte, A. Isidori, L. Marconi, Robust design of internal models by nonlinear regression, IFAC Proceedings Volumes 46 (23) (2013) 283-288.

[11] Z. Chen, J. Huang, Global robust output regulation for output feedback systems, IEEE Transactions on Automatic Control 50 (1) (2005) 117121.

[12] A. Serrani, A. Isidori, Global robust output regulation for a class of nonlinear systems, Systems \& Control Letters 39 (2) (2000) 133-139.

[13] A. Pavlov, L. Marconi, Incremental passivity and output regulation, Systems \& Control Letters 57 (5) (2008) 400-409.

[14] L. Liu, Z. Chen, J. Huang, Global disturbance rejection of lower triangular systems with an unknown linear exosystem, IEEE Transactions on Automatic Control 56 (7) (2011) 1690-1695.

[15] M. Bin, D. Astolfi, L. Marconi, Robust internal model design by nonlinear regression via low-power high-gain observers, in: 2016 IEEE 55th Conference on Decision and Control (CDC), IEEE, 4740-4745, 2016.

[16] M. Bin, D. Astolfi, L. Marconi, L. Praly, About robustness of internal model-based control for linear and nonlinear systems, in: 57th IEEE Conference on Decision and Control, 5397-5402, 2018.

[17] M. Bin, D. Astolfi, L. Marconi, About Robustness of Control Systems Embedding an Internal Model, arXiv preprint arXiv:2010.08794 .

[18] M. Bin, L. Marconi, A. R. Teel, Adaptive output regulation for linear systems via discrete-time identifiers, Automatica 105 (2019) 422-432.

[19] J. Ghosh, B. Paden, Nonlinear repetitive control, IEEE Transactions on Automatic Control 45 (5) (2000) 949-954.

[20] D. Astolfi, L. Praly, L. Marconi, Approximate regulation for nonlinear systems in presence of periodic disturbances, in: 54th IEEE Conference on Decision and Control, 7665-7670, 2015.

[21] P. Mattavelli, F. P. Marafao, Repetitive-based control for selective harmonic compensation in active power filters, IEEE Transactions on Industrial Electronics 51 (5) (2004) 1018-1024.

[22] G. Zarikian, A. Serrani, Harmonic disturbance rejection in tracking control of Euler-Lagrange systems: An external model approach, IEEE transactions on control systems technology 15 (1) (2006) 118-129.

[23] D. Astolfi, S. Marx, N. van de Wouw, Repetitive control design based on forwarding for nonlinear minimum-phase systems, Automatica 129 (2021) 109671.

[24] F. Mazenc, L. Praly, Adding integrations, saturated controls, and stabilization for feedforward systems, IEEE Transactions on Automatic Control 41 (11) (1996) 1559-1578. 
[25] D. Astolfi, L. Praly, Integral Action in Output Feedback for multi-input multi-output nonlinear systems, IEEE Transactions on Automatic Control 62 (4) (2017) 1559-1574

[26] B. Zitte, B. Hamroun, D. Astolfi, F. Couenne, Robust control of a class of bilinear systems by forwarding: Application to counter current heat exchanger, IFAC-PapersOnLine 53 (2) (2020) 11515-11520.

[27] T. Simon, M. Giaccagli, J.-F. Trégouët, D. Astolfi, V. Andrieu, X. Lin-Shi, H. Morel, Robust Output Set-Point Trackingfor a Power Flow Controller via Forwarding Design, in: 60th IEEE Conference on Decision and Control, IEEE, 2021.

[28] O. M. Grasselli, A. Isidori, F. Nicolo, Output regulation of a class of bilinear systems under constant disturbances, Automatica 15 (2) (1979) $189-195$.

[29] J. Kurzwel, On the inversion of Ljapunov's second theorem on stability of motion, AMS Translations Series 224 (1963) 19-77.

[30] D. Astolfi, L. Praly, L. Marconi, Nonlinear Robust Periodic Output Regulation of Minimum Phase Systems, Mathematics of Control, Signals, and Systems. Preprint arXiv:2109.06504.

[31] A. Isidori, L. Marconi, A. Serrani, Robust autonomous guidance: an internal model approach, Springer Science \& Business Media, 2012.

[32] A. Emami-Naeini, P. Van Dooren, Computation of zeros of linear multivariable systems, Automatica 18 (4) (1982) 415-430.

[33] L. Paunonen, Controller design for robust output regulation of regular linear systems, IEEE Transactions on Automatic Control 61 (10) (2015) 2974-2986.

[34] A. Terrand-Jeanne, V. Andrieu, V. D. S. Martins, C.-Z. Xu, Adding integral action for open-loop exponentially stable semigroups and application to boundary control of PDE systems, IEEE Transactions on Automatic Control 65 (11) (2019) 4481-4492.

[35] M. S. Benachour, V. Andrieu, L. Praly, H. Hammouri, Forwarding design with prescribed local behavior, IEEE Transactions on Automatic Control 58 (12) (2013) 3011-3023.

[36] D. Astolfi, L. Praly, L. Marconi, Francis-Wonham nonlinear viewpoint in output regulation of minimum phase systems, in: 11th IFAC Symposium on Nonlinear Control Systems, 2019.

[37] M. Giaccagli, D. Astolfi, V. Andrieu, L. Marconi, Sufficient Conditions for Output Reference Tracking for Nonlinear Systems: a Contractive Approach, in: 59th IEEE Conference on Decision and Control, IEEE, 4580 $4585,2020$.

[38] A. Krasnoselskii, P. P. Zabreiko, Geometrical methods of nonlinear analysis, vol. 263, Springer, 1984.

[39] T. Yoshizawa, Stability theory by Lyapunovs second method, vol. 14, The mathematical Society of Japan, 1966.

[40] R. K. Miller, A. N. Michel, Ordinary differential equations, Academic Press, 1982.

[41] J. Hale, Ordinary differential equations, Krieger Publishing Company, 1980.

[42] A. Isidori, Nonlinear control systems II, Springer Science \& Business Media, 1999. 